\title{
Broad band impedance measurements on the electron storage ring ELETTRA
}

\author{
Emanuel Karantzoulis, Victor Smaluk, and Lidia Tosi \\ Sincrotrone Trieste, Trieste, Italy \\ (Received 1 October 2002; published 26 March 2003)
}

\begin{abstract}
In the design of the vacuum chamber of a storage ring, special care has to be taken to keep the impedance as low as possible, in order to not degrade the quality of the circulating beam. Since the start of the commissioning of the Italian synchrotron light source ELETTRA in 1993, several low gap insertion vacuum chambers with internal aperture of $14 \mathrm{~mm}$ have been installed into the storage ring. Monitoring of their individual impedances and effects has been carried out with measurements on the electron beam after each new installation. The subsequent evolutions of the total longitudinal and transverse impedances are presented in this paper. Furthermore, in contradiction with the foreseen single-bunch effect, noticeable excitations of particular narrow band longitudinal coupled multibunch instabilities as a function of the position of the blades of the vertical scraper have been observed. In order to achieve a deeper insight of the phenomenon, an exhaustive characterization of both the longitudinal and transverse impedances of the scraper has been carried out.
\end{abstract}

DOI: 10.1103/PhysRevSTAB.6.030703

PACS numbers: 29.20.-c, 29.27.Bd

\section{INTRODUCTION}

ELETTRA is the Italian third generation synchrotron light source generating photons, mainly provided by insertion devices, in the very ultraviolet to the soft x-ray regions. While the injector is a $1.0 \mathrm{GeV}$ linac, its operation energies are 2.0 and $2.4 \mathrm{GeV}$ for, respectively, $75 \%$ and $25 \%$ of the user time. As for all third generation light sources, the high brilliance and quality of the photons are guaranteed by the stringent requirements on the electron beam parameters, its high stability, and the maximum achievable beam current. Table I shows the design values for ELETTRA.

Most of these parameters depend strongly on the impedance of the storage ring. In fact, any instability driven by a too large narrow or broad band impedance would increase the energy spread as well as the effective emittance, degrading the quality of the light. The impedance status is reflected greatly by the maximum achievable stored current and during the first year of commissioning it was possible to store more than $60 \mathrm{~mA}$ in a single bunch and $700 \mathrm{~mA}$ in a multibunch with an $80 \%$ filling, exceeding by a large extent the design values in both cases. For the multibunch case, no injection saturation effects were

TABLE I. ELETTRA design parameters at $2 \mathrm{CeV}$.

\begin{tabular}{lc}
\hline \hline \multicolumn{1}{c}{ Parameter } & Value \\
\hline Circumference $(\mathrm{m})$ & 259.2 \\
Beam current, multibunch mode $(\mathrm{mA})$ & 400 \\
Beam current, single-bunch mode $(\mathrm{mA})$ & 9.8 \\
Natural energy spread, rms & $8 \times 10^{-4}$ \\
Natural bunch length, rms $(\mathrm{mm})$ & 5.4 \\
Natural emittance $(\pi \mathrm{mrad})$ & $7 \times 10^{-9}$ \\
Beam size at ID centers, horizontal/vertical $(\mu \mathrm{m})$ & $241 / 15$ \\
Betatron tunes, horizontal/vertical & $14.3 / 8.2$ \\
\hline \hline
\end{tabular}

030703-1

$1098-4402 / 03 / 6(3) / 030703(8) \$ 20.00$ noticed and the limit was imposed only to not damage some diagnostic instrumentations.

Apart from the rf cavities which are four single cells with a smooth bell shape, the other important contributor to the overall impedance is the vacuum chamber. Its contribution comes from both its shape and material as well as from its discontinuities such as holes, steps, connections, etc., and the chamber was carefully designed so as to keep its influence on the beam as low as possible. During commissioning in 1993 the vacuum chamber at the 11 straight sections to be used for the insertion devices was rhomboidal made of stainless steel with internal dimensions $82 \times 53 \mathrm{~mm}$. In 1994 four straight rhomboidal chambers where replaced by rectangular ones of the same material with internal dimensions $70 \times 20 \mathrm{~mm}$. Since 1997 (see Table II) straight section chambers were replaced with narrow gap ones with dimensions $82 \times$ $14.8 \mathrm{~mm}$ for the stainless steel and $81 \times 14 \mathrm{~mm}$ for the aluminum ones.

TABLE II. Dates of the installations of low gap straight sections (ID) and bending magnet (BM) chambers.

\begin{tabular}{ccc}
\hline \hline \multicolumn{1}{c}{ Date } & Section & Material \\
\hline Sep. 1997 & ID $4(4.0 \mathrm{~m})$ & Stainless steel \\
Oct. 1998 & ID $9(4.8 \mathrm{~m})$ & Aluminum \\
Oct. 1998 & BM 9.2 & Aluminum \\
Aug. 1999 & ID $1(4.8 \mathrm{~m})$ & Aluminum \\
Aug. 1999 & BM 1.2 & Aluminum \\
Sep. 1999 & BM 1.1 & Aluminum \\
Jan. 2000 & ID $2(4.8 \mathrm{~m})$ & Aluminum \\
Apr. 2000 & ID $8(4.8 \mathrm{~m})$ & Aluminum \\
Apr. 2000 & BM 8.1 & Aluminum \\
Jan. 2002 & ID $10(4.8 \mathrm{~m})$ & NEG coated aluminum \\
\hline \hline
\end{tabular}


Impedance budget calculations [1] using mostly analytic formulas estimated that the broad band longitudinal and transverse impedances would have been $\leq 0.7 \Omega$ and $\leq 200 \mathrm{k} \Omega / \mathrm{m}$, respectively, with an estimated cutoff frequency of about $2.3 \mathrm{GHz}$. The predictions worked quite well since single-bunch measurements showed that the broad band impedances were $\sim 0.5 \Omega$ and $\sim 130 \mathrm{k} \Omega / \mathrm{m}$. These values confirm also the theoretically predicted high current thresholds of ELETTRA confirming that the budget analysis can be reliable. The instability situation at the end of the first year of commissioning was as follows. The resistive wall-type instability was not observed. The closest unstable frequency due to the fractional part of the vertical tune of 0.2 is $0.8 f_{0}=0.925 \mathrm{MHz}$ with $f_{0}=1.156 \mathrm{MHz}$ as the revolution frequency. At this frequency the pessimistic impedance obtained from assuming all the machine with the chamber dimensions as in the insertion device sections was $800 \mathrm{k} \Omega / \mathrm{m}$. For this value, with $300 \mathrm{~mA}$ in multibunch at $20 \mathrm{GeV}$, the resistive wall growth rate is $\sim 3 \mathrm{~ms}$ corresponding to a coherent frequency shift of $\sim 300 \mathrm{~Hz}$. For this to be Landau damped the incoherent betatron frequency spread must be at least $600 \mathrm{~Hz}$ which is well inside the measured spread of $\leq 1 \mathrm{kHz}$. Multibunch instabilities driven by the higher order modes (HOMs) of the rf cavities existed as predicted $[2,3]$. Careful tuning of the cavity volume via temperature control shifts the HOMs away from the beam harmonics and a complete instability free condition was achieved at $150 \mathrm{~mA}$ [4]. As for the transverse mode coupling, the threshold was predicted at $40 \mathrm{~mA} /$ bunch but was not seen. Simple threshold estimations with the actual impedance set the threshold at $58 \mathrm{~mA}$ for $1 \mathrm{GeV}$ but $65 \mathrm{~mA}$ were injected without saturation. However above $50 \mathrm{~mA}$ the beam was blown up and throbbing. Regarding the head-tail instabilities, the mode $m=0$ was stable for small vertical positive chromaticity $\xi_{y}=0.1$. At higher currents the modes $m=-1,1$ were seen but no mode merging occurred. With a slightly negative chromaticity $\xi_{y}=-0.5$ the mode $m=0$ threshold was found at $\sim 22 \mathrm{~mA}$ above which the beam blew up vertically and oscillated. This threshold was theoretically expected since using the measured impedance data the necessary Landau damping frequency full spread should have been at about $400 \mathrm{~Hz}$, whereas the "natural" tune spread available $\Delta \nu / \nu$ is $\sim 6 \times 10^{-6}$ yielding a frequency spread of $357 \mathrm{~Hz}$.

Because of the scarce interest of users in single bunch, the lack of special instrumentation, the low impedance, the reliability of the model, and no serious instability problems, no beam excitation measurements to distinguish the real and imaginary parts of the impedances or other additional measurements were performed at that time. ELETTRA however, never stopped evolving and since 1997 the insertion device straight section vacuum chambers are being replaced with vertically low gap $(14 \mathrm{~mm})$ ones, mostly made of aluminum, as well as some bending magnet (BM) vacuum chambers made of aluminum with a modified internal aperture for photon extraction, as seen in Table II.

Many measurements have been performed to monitor eventual impedance changes. With the help of the transverse multibunch feedback [5] (TMBF) instrumentation and a streak camera it was possible to measure the resistive and the reactive parts of both the transverse and longitudinal impedances. The measurements, the results, and their implications are presented and discussed in this paper.

Furthermore the low gap vacuum chambers limit the physical aperture and increase the possibility of beam loss at their location. In order to provide a radiation safe condition for users, the vertical scraper will be used as a shield in case of a beam loss. A series of experiments is performed to understand the impedance behavior of this object, very important for radiation safe operation, and at the same time to ensure that it does not deteriorate the beam quality.

\section{TOTAL LONGITUDINAL IMPEDANCE}

An electron beam passing through any irregularity of a vacuum chamber loses energy. The total energy loss $\Delta E$ is proportional to the square of the beam charge $q$ [6]:

$$
\Delta E=-k_{\|} q^{2} .
$$

The proportionality factor $k_{\|}$is called the longitudinal loss factor. It depends both on the vacuum chamber structure characterized by the wake potential $W_{\|}$and on the particle distribution $\rho$ :

$$
k_{\|}=\int_{-\infty}^{\infty} W_{\|}(t) \rho(t) d t,
$$

or, in the frequency domain:

$$
k_{\|}=\frac{1}{\pi} \int_{0}^{\infty} \Re Z_{\|}(\omega)|\tilde{\rho}(\omega)|^{2} d \omega,
$$

where $Z_{\|}(\omega)$ is the longitudinal broad band impedance of the vacuum chamber and $\tilde{\rho}(\omega)$ is the Fourier transform of the particle distribution $\rho(t)$.

The total longitudinal loss factor of the ring was measured. The measurement method is based on the indirect measurement of the beam energy loss (1) using the standard system of beam position monitors (BPM). The energy loss $\Delta E$ results in a closed orbit deviation in the dispersive regions of the storage ring:

$$
\Delta x(z)=\eta(z) \frac{\Delta E}{E},
$$

where $\eta(z)$ is the dispersion and $E$ is the total beam energy. Thus, the longitudinal loss factor $k_{\|}$can be estimated by measuring the horizontal closed orbit deviation with the beam intensity. Figure 1 shows the measured 


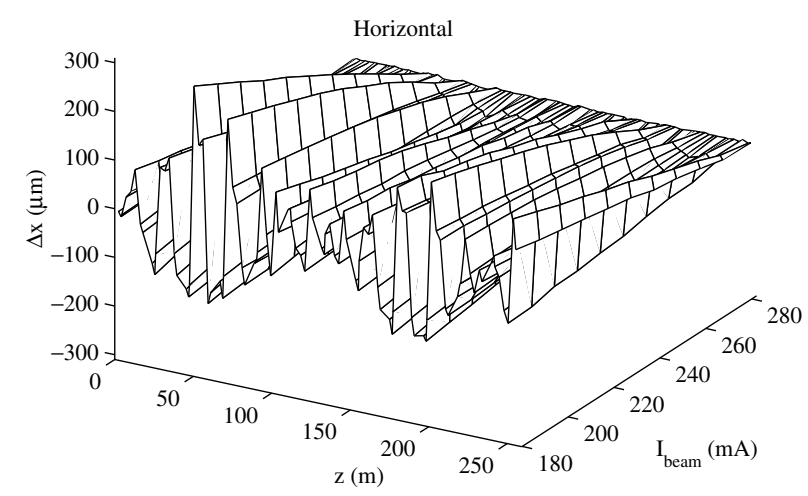

FIG. 1. Horizontal orbit deviation versus beam current.

horizontal orbit deviation at $2.0 \mathrm{GeV}$ as a function of beam current.

Before the measurement, the orbit was corrected globally to minimize the influence of the transverse impedance. The reference orbit was taken to be the one at the beam current of $280 \mathrm{~mA}$ and orbit deviations were measured at several decreasing beam currents. There are two known thermal effects, which can introduce a systematic error into the measurement: the thermal motion of the vacuum chamber and, therefore, of the BPMs after ramping from the injection energy of 0.9 to $2.0 \mathrm{GeV}$, and the thermal motion due to change of beam heating during the beam current variation. To avoid an influence of these effects, the measurement was done $1 \mathrm{~h}$ after the ramping, when the storage ring temperature was stabilized, and in a short time span.

The BPM current-dependent nonlinearity influences to the same order both horizontal and vertical planes. So, taking the vertical orbit deviation, which is less than $10 \%$ of the horizontal one and assuming zero dispersion in the vertical plane, one concludes that the BPM errors are less than $10 \%$ to the measured values. This is further demonstrated observing the dispersionlike shape of the horizontal orbit deviation, while the vertical one looks like a noise.

Figure 2 shows the measured horizontal and vertical dispersions. By comparing the figures it can be observed that the horizontal orbit deviation is proportional to the dispersion and, in particular, the slow wave in both measurements is noticed. While the vertical orbit deviation was found to be negligible because of the small vertical dispersion, the horizontal one grows up to about $200 \mu \mathrm{m}$. Taking the orbit deviation values at the points with a high dispersion to increase the accuracy, the coherent energy loss proportional to the beam current was calculated. The measurement result is shown in Fig. 3. Thus the longitudinal loss factor of the ring is $12.6 \pm 2.8 \mathrm{~V} / \mathrm{pC}$, and the real part of the total longitudinal impedance is $0.12 \pm$ $0.03 \Omega$ for $5.4 \mathrm{~mm}$ of $\mathrm{rms}$ bunch length.

As for the total module of the effective longitudinal impedance

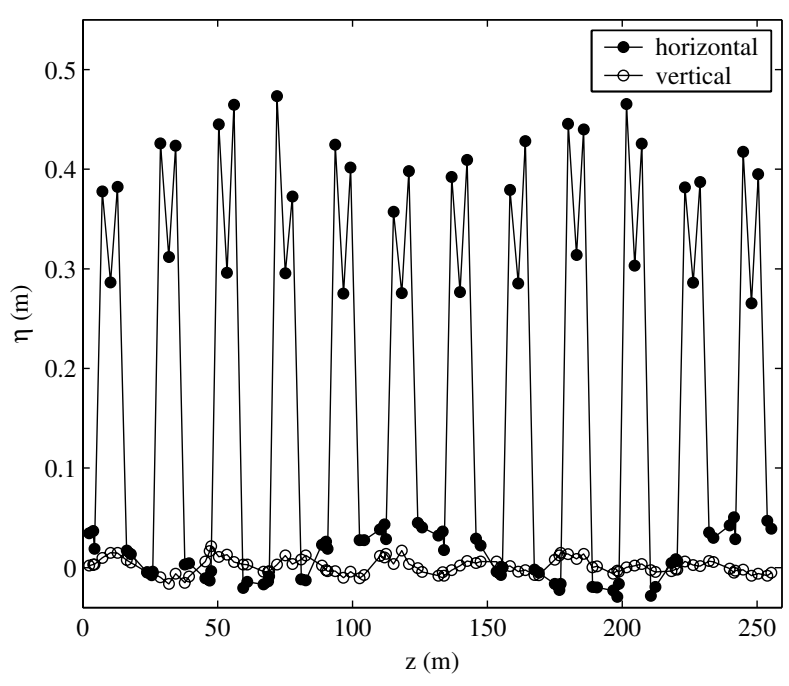

FIG. 2. Measured dispersion.

$$
\left|\frac{Z_{\|}}{n}\right|^{\text {eff }}=2\left(\frac{\sigma_{z} \omega_{c}}{c}\right)^{2}\left|\frac{Z_{\|}}{n}\right|
$$

where $\sigma_{z}$ is the rms bunch length and $\omega_{c}$ the cyclic cutoff frequency, bunch length measurements in single-bunch mode have been performed (see Fig. 4) since the beginning using fast oscilloscopes, photodiodes, and finally a streak camera. The effective longitudinal broad band impedance can be obtained using the microwave stability limit given by the well-known formula [7]:

$$
\left|\frac{Z_{\|}}{n}\right|_{\mathrm{BBR}}^{\text {eff }} \leq \frac{|\alpha-1 / \gamma| \beta^{2}(E / e)}{I_{p}}\left(\frac{\Delta p}{p}\right)_{\mathrm{FWHM}}^{2},
$$

where $\alpha$ is the momentum compaction factor, $\gamma=$ $\left(E / m_{e} c^{2}\right), \beta=\sqrt{1-1 / \gamma^{2}}$, and $I_{p}$ is the peak bunch current defined for a Gaussian bunch as

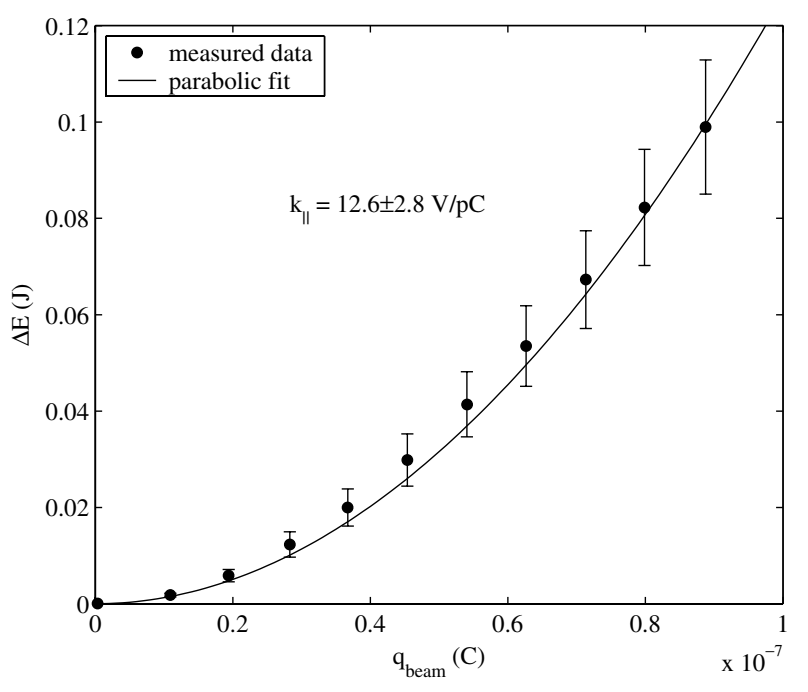

FIG. 3. Total longitudinal energy loss versus beam charge. 


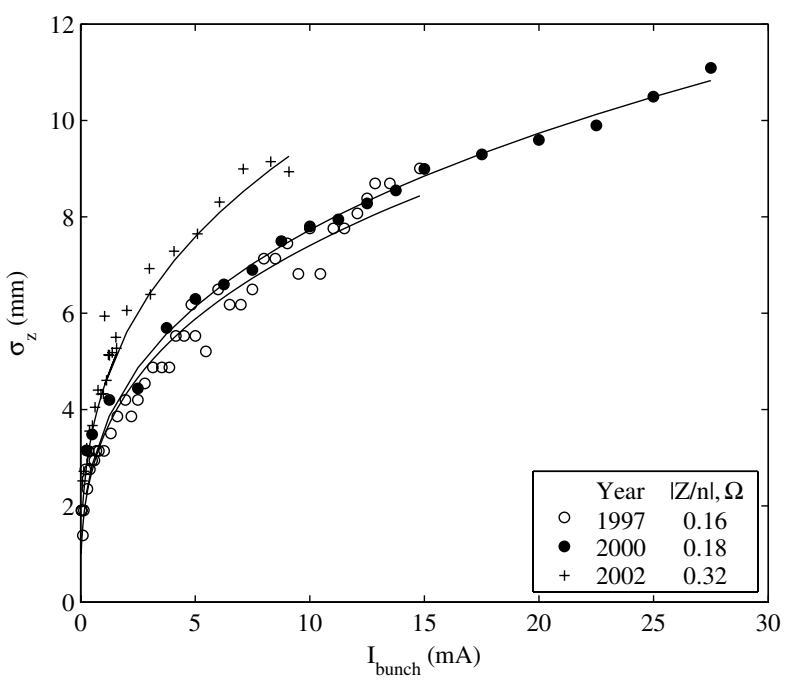

FIG. 4. Bunch lengthening versus current.

$$
I_{p}=\frac{\sqrt{2} \bar{\pi} R}{\sigma_{z}} I_{b}
$$

For electron machines, where $\gamma \gg 1$ and $\beta \simeq 1$, Eq. (6) can be written in a more convenient form as [1]

$$
\left|\frac{Z_{\|}}{n}\right|_{\mathrm{BBR}}^{\text {eff }} \leq \frac{8 \ln 2}{2 \pi} \frac{k V_{\mathrm{rf}} \cos \phi_{s}}{\sqrt{2 \pi} I_{b}}\left(\frac{\sigma_{z}}{R}\right)^{3},
$$

where $k$ is the $\mathrm{rf}$ harmonic number, $V_{\mathrm{rf}}$ is the rf voltage, $\phi_{s}$ is the rf equilibrium phase, $I_{b}$ is the average bunch current, and $R$ is the average machine radius. Thus the impedance can be estimated directly from measurements of the bunch length, by plotting the bunch length versus the $1 / 3$ power of the current which for the turbulence regime should show a linear dependence. The slope of the linear fit is proportionally related to the effective impedance whereas the cutoff frequency induces a quadratic dependence for the same bunch length [see Eq. (5)].

As can be rated from Fig. 4, measurements until the year 2000 do not indicate clear changes in the bunch length for the same current. The last measurements were performed with the streak camera and they indicate an effective broad band longitudinal impedance value of $0.32 \Omega$, i.e., inside the determined threshold of the impedance budget analysis. This value however appears almost double from its initial value obtained from measurements with other instruments. Since the biggest contributors of the longitudinal machine impedance are the rf cavities, this inconsistency may be due to the different measurement methods and accuracies rather than to a real doubling of the longitudinal impedance. On the other hand, it can be also attributed to the increase of the cutoff frequency estimated to be now at $3.5 \mathrm{GHz}$. One can however conclude, combining the longitudinal resistive and total impedance results that the longitudinal effective impedance lies between $0.2-0.3 \Omega$.

\section{TOTAL TRANSVERSE IMPEDANCE}

Both the resistive $\mathfrak{R} Z_{\perp}$ and the reactive $\Im Z_{\perp}$ components of the transverse impedance have been measured. Beam interaction with a resistive transverse impedance results in the well-studied head-tail instability characterized by the head-tail phase [8]:

$$
\chi=\frac{2 \pi \nu f_{0} \xi \sigma_{z}}{c \alpha}
$$

where $\nu$ is the betatron tune, $f_{0}$ the revolution frequency, $\xi$ the chromaticity, $\sigma_{z}$ the rms of the bunch length, $c$ the speed of light, and $\alpha$ the momentum compaction factor. If the head-tail phase $\chi$ is not much less than unity, the growth rate of the betatron oscillations is

$$
\tau_{ \pm}^{-1}=\mp \frac{I_{b} c^{2} \mathfrak{I} f(2 \chi)}{32 \pi^{2}(E / e) \nu f_{0} b} \Re Z_{\perp},
$$

where $I_{b}$ is the bunch current, $E$ is the particle energy, $b$ is the average vacuum chamber radius, and $f(2 \chi)$ is the complex function $f(u)=\int_{0}^{\pi} e^{i u \sin x} d x$ of the head-tail phase $\chi$. Coherent (center-of-mass) motion is characterized by $\tau_{+}$and the incoherent (beam size) motion by $\tau_{-}$. The "+" mode is damped if $\xi / \alpha>0$ and antidamped if $\xi / \alpha<0$, whereas for the "- " mode the mechanism is vice versa. So, when $\xi$ is made slightly positive and a coherent betatron oscillation is excited by a kicker, $\mathfrak{R} Z_{\perp}$ can be evaluated by measuring the damping time $\tau_{+}$of the oscillation.

For various beam currents, the damping rates were estimated by the analysis of the turn-by-turn beam positions measured with the TMBF system. Whereas coherent horizontal betatron oscillations were excited by the injection kickers, resonance excitation was used to produce vertical oscillations.

The damping rates with the beam current are shown in Fig. 5. On the basis of these measurements, the resistive

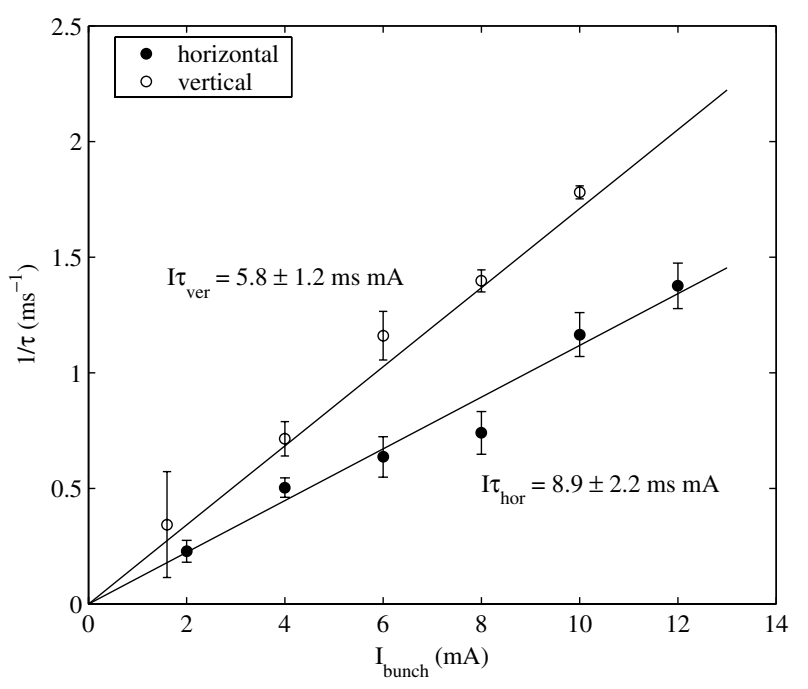

FIG. 5. Coherent head-tail damping. 
part of the transverse impedance results to be $\mathfrak{R} Z_{\perp}=$ $0.16 \pm 0.05 \mathrm{M} \Omega / \mathrm{m}$.

The reactive transverse impedance $\Im Z_{\perp}$ produces a coherent current-dependent shift of a betatron tune as

$$
\Delta \nu=-\frac{I_{b} R}{4 \pi \sigma_{z}(E / e)} \sum_{i} \beta_{i} \Im Z_{\perp i} .
$$

Here $\beta_{i}$ is the beta function of the $i$ th (low gap vacuum chamber) impedance location.

The detuning of the coherent vertical betatron oscillation with single-bunch current for the rigid mode was measured after each insertion device chamber installation using the spectrum analyzer of the tune measurement system with a precision of $250 \mathrm{~Hz}$. The results are summarized in Fig. 6.

It is quite interesting to observe that the vertical effective impedance increases proportionally with the number of low gap chambers, i.e., each chamber contributes on the average $0.12 \pm 0.02 \mathrm{kHz} / \mathrm{mA}$ (with the statistical $\sigma=0.03$ ) to the original $0.12 \mathrm{kHz} / \mathrm{mA}$. With the installation of the last nonevaporable getter (NEG) aluminum chamber it has been measured a rather unexpected increase of about $0.24 \pm 0.02 \mathrm{kHz} / \mathrm{mA}$ which lies at $4 \sigma$ from the previously established average and 6 times above the measurement error. The previous maximum of $0.17 \mathrm{kHz} / \mathrm{mA}$ occurred with the installation of $\mathrm{S} 2.2$ (February 2000) and was $3 \sigma$ out of the then established average (0.11 with $\sigma=0.02$ ) and the low gap BPMs were held as responsible for the small deviation. At least one more set of measurements needs to be performed with the installation of an additional identical NEG coated chamber later this year in order to be able to evaluate whether the observed increase is an effect due to the NEG coating.

In May 2001 before the installation of the NEG coated chamber precise measurements of both the horizontal and vertical coherent tune shifts were performed using the

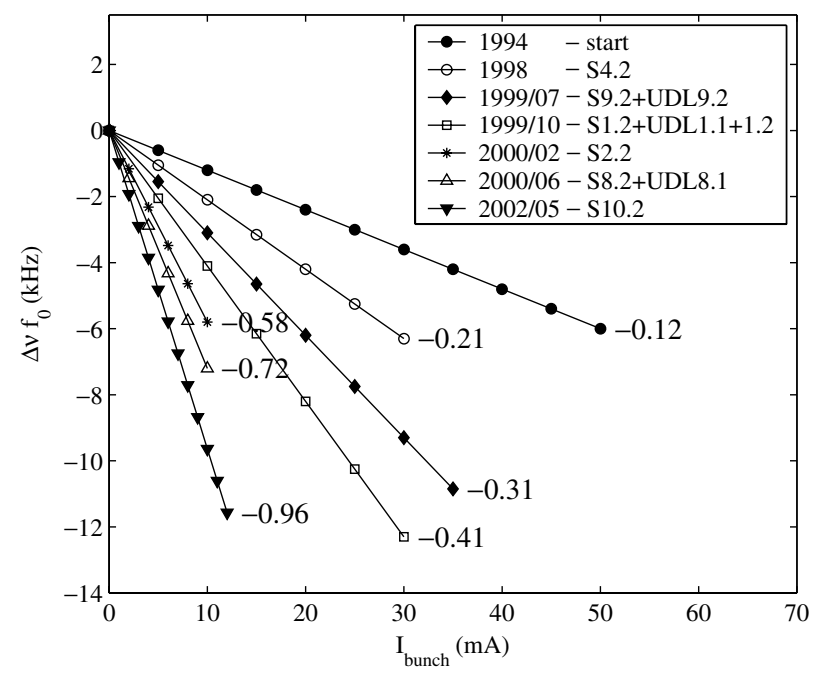

FIG. 6. Vertical coherent tune shift evolution.

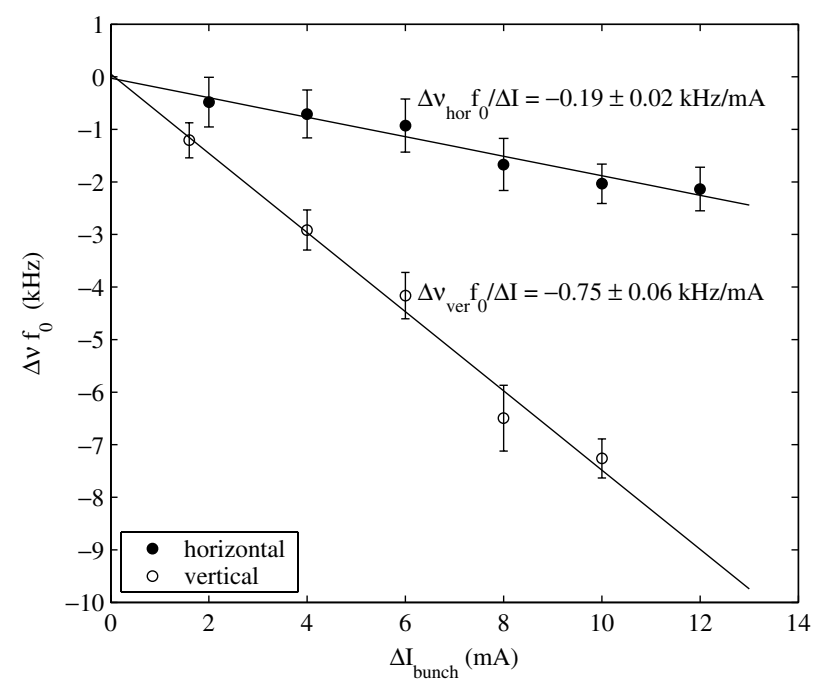

FIG. 7. Horizontal and vertical coherent tune shifts.

TMBF system. The results are shown in Fig. 7. It must be noted that for the first time horizontal tune shifts were observed due to the higher precision of the tune measurement method which is better than $10^{-4} f_{0}=115 \mathrm{~Hz}$. The measured vertical betatron frequency shift $-0.75 \pm$ $0.04 \mathrm{kHz} / \mathrm{mA}$ was found much greater than the horizontal one $-0.19 \pm 0.02 \mathrm{kHz} / \mathrm{mA}$, due to the reduced physical aperture in the vertical plane. The total reactive transverse impedance, calculated from these measurements and assuming a bunch length as $\sigma_{z}=5.4 \mathrm{~mm}$, is $\Im Z_{\perp}=0.3 \pm 0.04 \mathrm{M} \Omega / \mathrm{m}$. Taking also the latest data into account the value of the reactive vertical impedance should be now about $0.5 \mathrm{M} \Omega / \mathrm{m}$.

\section{SCRAPER IMPEDANCE}

When starting the operation of the transverse multibunch feedback system during user dedicated beam time, questions arose regarding the protection of the vacuum chamber against overheating due to insertion device photons hitting the chamber in case of a feedback malfunction. For this purpose the vertical scraper was thought to be used as a means to reduce the beam intensity in case large vertical betatron oscillations were present. However, during first measurements, it was observed that the scraper induced longitudinal coupled multibunch modes as a function of the blades' positions when the latter were below $\pm 8 \mathrm{~mm}$. The modes were found to be strong enough to suppress the transverse ones and at a scraper setting of $\pm 7 \mathrm{~mm}$ there was an enhancement of the excitation of a known cavity mode, which could be reduced in amplitude by acting on the cavity.

In ELETTRA the vertical scraper is discontinuous and is composed of two $1 \mathrm{~cm}$ in diameter rods positioned at $\pm 25 \mathrm{~mm}$ when it is open. Analytical formulas for the impedance of such a geometry do not exist and evaluation 
of its behavior has been performed using the electron beam.

\section{A. Longitudinal loss factor}

In order to estimate the contribution of the vertical scraper to the total longitudinal loss factor, the orbit deviation with various scraper apertures was measured. The measurement was carried out at a beam current of $195 \mathrm{~mA}$. In order to avoid transverse impedance effects, the beam position at the scraper was corrected to the geometrical center of the scraper and the scraper blades were moved symmetrically with respect to the center. Figure 8 shows the measured horizontal orbit deviation.

The horizontal orbit deviation looks similar to the one shown in Fig. 1, although with an order of magnitude less in amplitude and much more noisy. The vertical one was found to be within the measurement error.

Figure 9 shows the longitudinal loss factor caused by the scraper. In order to compare the order of magnitudes, the loss factors calculated for the axially symmetric

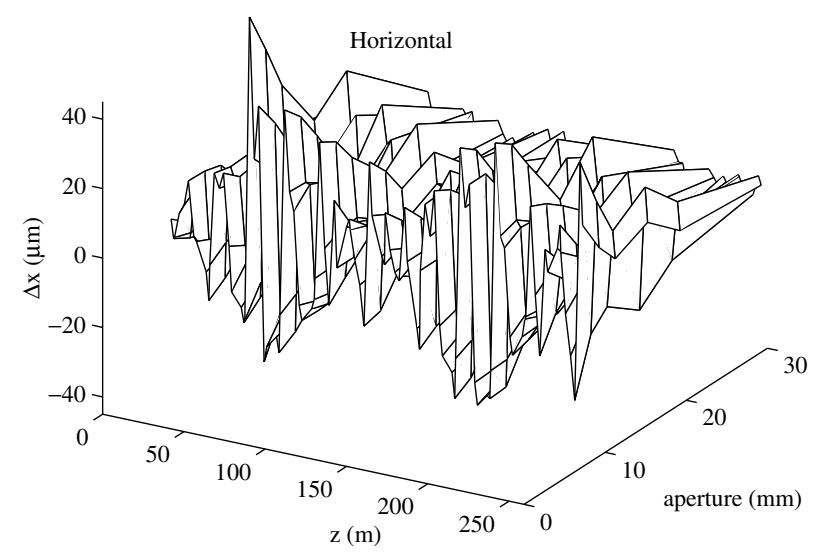

FIG. 8. Horizontal orbit deviation versus scraper aperture.

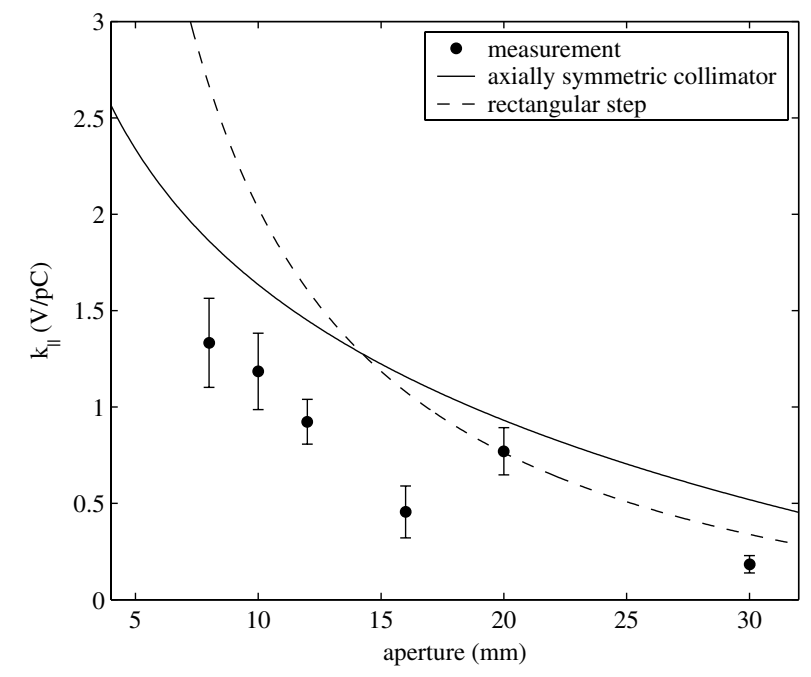

FIG. 9. Longitudinal loss factor caused by the scraper. collimator [6] and for the rectangular step [1] of the same size are shown. Thus, as a result of the measurement, it can be concluded that the scraper contribution to the total longitudinal loss factor is around $6 \%-10 \%$ when the scraper aperture is $8-10 \mathrm{~mm}$.

Measurements of the imaginary part of the longitudinal impedance, which would have given a deeper insight on the excitations of the longitudinal coupled multibunch instabilities, have also been tried. The results, however, were found to be of the order of the measurement precision. It can be nevertheless expected that it is of the same order as the resistive part.

\section{B. Reactive transverse impedance}

To estimate the scraper contribution to the total reactive transverse impedance of the storage ring, two measurement methods were used. The first method is based on the direct measurement of the coherent tune shift (11). The total machine impedance was estimated by the measurement of the current-dependent tune shift $\Delta \nu / \Delta I_{b}$ and the contribution of the scraper by the measurement of the tune shift as a function of the scraper blades' position at constant current. Figure 10 shows the results, but the accuracy of the method is limited by the resolution of the tune measurement system which is insufficient to measure such a small effect.

To increase the accuracy, a variant of the bump method [9] was used with the scraper slit vertically shifted instead of the orbit bump. As shown in [10], the method with some limitations can be used for such an asymmetrical structure as the scraper. The closed orbit deviation proportional to the impedance and to the scraper blade position was measured using the BPM system:

$$
y(z)=\frac{\sqrt{\beta_{s} \beta(z)} \cos \left(\pi \nu-\left|\phi_{s}-\phi(z)\right|\right)}{2(E / e) \sin \pi \nu} I_{p} \Im Z_{\perp} \Delta y,
$$

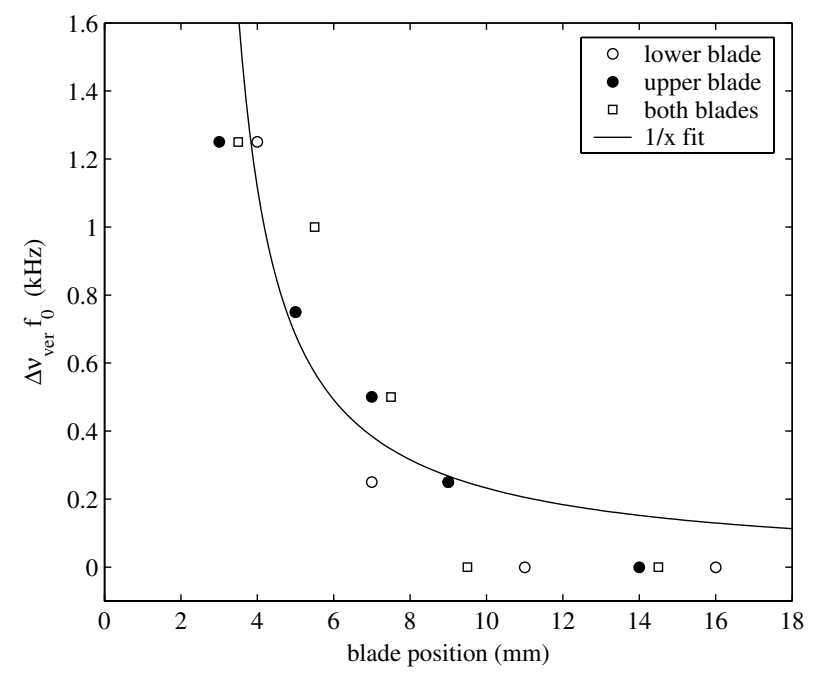

FIG. 10. Tune shift versus scraper blades' position. 


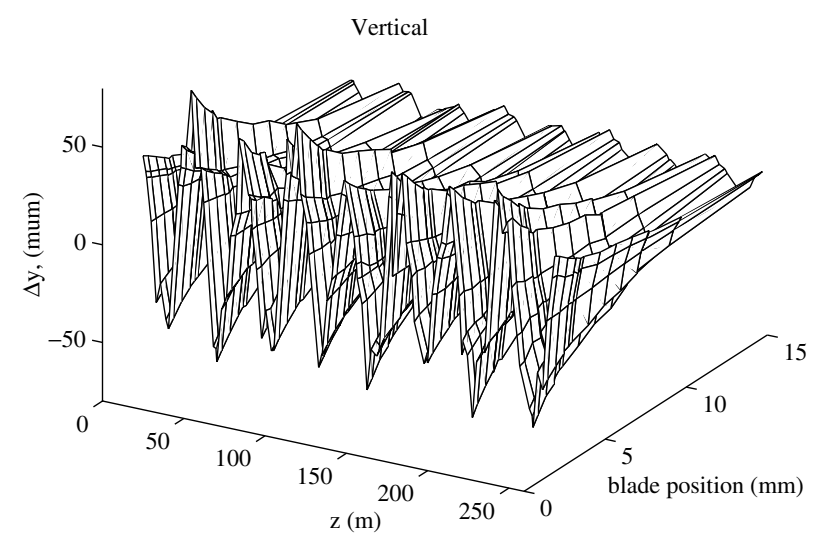

FIG. 11. Vertical orbit deviation versus scraper blade position.

where $I_{p}$ is the peak bunch current, $\Delta y=y_{b 0}-y_{b}$, and $y_{b 0}$ and $y_{b}$ are the open and closed scraper blade positions, respectively. Figure 11 shows the vertical orbit deviation as a function of the scraper blade position. The horizontal one is negligible.

The corresponding measured scraper impedance is shown in Fig. 12.

The measured data are approximated well by the function describing a small-angle rectangular collimator [11]:

$$
Z_{\perp}=-\frac{i Z_{0} h}{2} \int \frac{\left(b^{\prime}\right)^{2}}{b^{3}}
$$

where $Z_{0}=120 \pi \Omega$ is the free space impedance, $h$ in our case is the scraper rod diameter, and $b(z)$ is the half-gap. In order to model $b(z)$, an exponential function

$$
b(z)=d e^{-(3 z / 2 d)}
$$

was chosen as a best continuous approximation to the situation (see Fig. 13). With this function, a simple formula for the impedance is obtained as

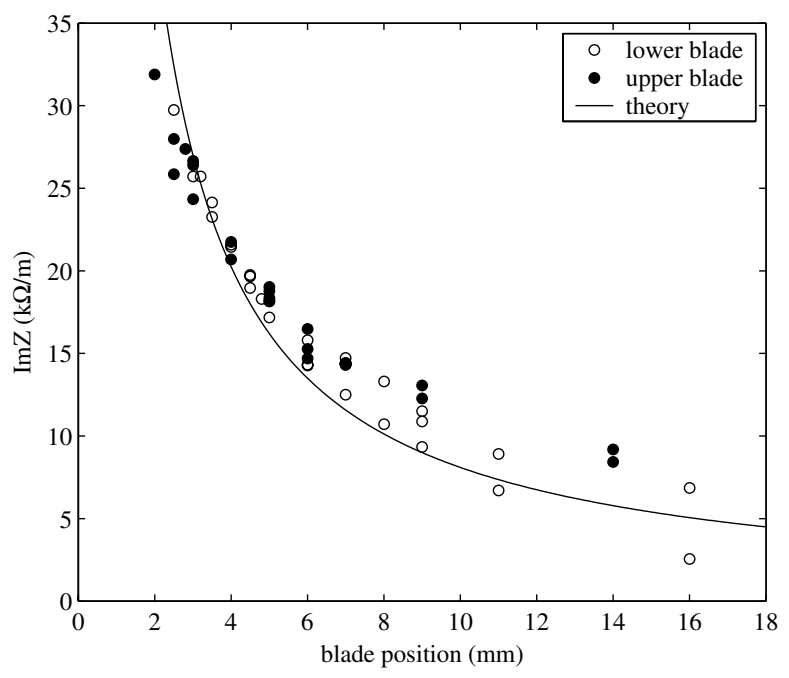

FIG. 12. Scraper vertical impedance.

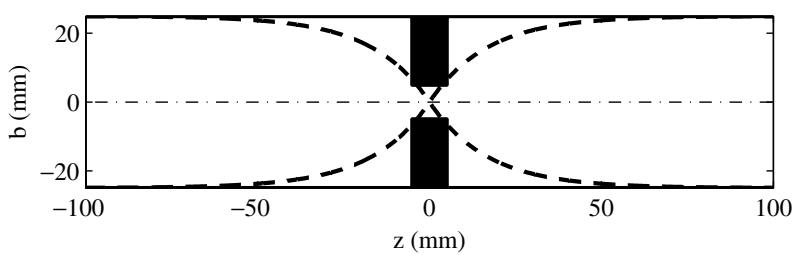

FIG. 13. Smooth approximation of the scraper profile.

$$
Z_{\perp}=\frac{3}{4} \frac{Z_{0} h}{d}\left(\frac{1}{b}-\frac{1}{d}\right)
$$

where $d=25 \mathrm{~mm}$ is the maximal half-gap. It can be observed that the formula fits the data very well.

Thus at the $\pm 5 \mathrm{~mm}$ gap the transverse impedance of the scraper is about $10 \%$ of the total impedance or about $30 \mathrm{k} \Omega / \mathrm{m}$.

\section{CONCLUSIONS}

As ELETTRA is diverging from the "perfect" machine [i.e., no narrow gap insertion device (ID) chambers], its impedance is increasing. The total longitudinal effective impedance is now $0.2-0.3 \Omega$, while the vertical and horizontal ones are about $0.5 \mathrm{M} \Omega / \mathrm{m}$ and less than $0.1 \mathrm{M} \Omega / \mathrm{m}$, respectively. From the longitudinal and transverse impedance measurements one may conclude that the present value of the cutoff frequency that better fits all the data has increased from the initial $2.3 \mathrm{GHz}$ to about $3.5 \mathrm{GHz}$.

It is expected that by the end of all the insertion device chamber installations the transverse impedance will exceed $0.5 \mathrm{M} \Omega / \mathrm{m}$ which means $\Delta \nu / \Delta I>1 \mathrm{kHz} / \mathrm{mA}$. In this case no more than $8 \mathrm{~mA} /$ bunch will be stored unless one starts increasing the chromaticity.

With these new developments the present instability situation is as follows. Resistive wall instability is still not clearly observed. After the first installation of the stainless steel low gap chamber both horizontal and vertical resistive wall-like signals were noticed but could not be clearly identified as such. Adding all other chambers the situation did not change, nor an enhancement of the signals noticed. Detuning of the harmonic sextupole was sufficient to give the necessary damping and the effect was canceled $[12,13]$. The resistive wall impedance at $0.8 f_{0}=0.93 \mathrm{MHz}$ assuming an equivalent chamber radius of $10 \mathrm{~mm}$ for the whole ring made of aluminum is $1.3 \mathrm{M} \Omega / \mathrm{m}$. Adding the $0.8 \mathrm{M} \Omega / \mathrm{m}$ found previously, it is safe to assume a total maximum impedance of $2 \mathrm{M} \Omega / \mathrm{m}$ which will give for $300 \mathrm{~mA}$ at $2 \mathrm{GeV}$ a minimum growth time of $\sim 1.4 \mathrm{~ms}$ corresponding to a coherent frequency shift of $\sim 700 \mathrm{~Hz}$. For that to be Landau damped the incoherent betatron frequency spread must be at least $1.4 \mathrm{kHz}$. Longitudinal multibunch instabilities did not change their behavior. Concerning transverse mode coupling, the mode $m=0$ and $m=-1$ merging at $40 \mathrm{~mA} /$ bunch was seen with nearly zero chromaticities 
[14]. The head-tail instabilities result in the single-bunch current limit and with slightly negative chromaticity no more than $12 \mathrm{~mA}$ can be stored.

The scraper contributes about $10 \%$ to the total impedance when it is closed down to $\pm 5 \mathrm{~mm}$. Extraction of the function that fits the measurement data shows that the vertical rod scraper behaves more like a tapered device rather than a step scraper. In fact the data fit very well to a function of the type as $\frac{1}{x}$ rather than one as $\frac{1}{x^{2}}$ which is expected by a step device. Furthermore unforeseen longitudinal multibunch instabilities which depend on the blades' positions when both are closed have been observed. The measured value of the longitudinal impedance is not sufficient to explain these excitations. Efforts are presently being put into understanding their behavior which is thought to be due to an accidental cavity created by the rod and some antichamber [13]. This finding had an important impact since for the moment the design and development of a tapered scraper has been stopped until the cause has been understood.

[1] E. Karantzoulis, Sincrotrone Trieste Report No. ST/ M-TN-90/14, 1990.

[2] E. Karantzoulis, C. J. Bocchetta, F. Iazzourene, R. Nagaoka, L. Tosi, R. P. Walker, and A. Wrulich, in Proceedings of the EPAC-94, London (World Scientific, Singapore, 1994).

[3] M. Svandrlik, G. D'Auria, A. Fabris, E. Karantzoulis, A. Massarotti, C. Pasotti, and C. Rossi, in Proceedings of the EPAC-94, London (Ref. [2]).
[4] E. Karantzoulis, C. J. Bocchetta, A. Fabris, F. Iazzourene, M. Svandrlik, L. Tosi, and R. P. Walker, in Proceedings of the EPAC-98, Stockholm (IOP, Bristol, 1998).

[5] D. Bulfone, C. J. Bocchetta, R. Bressanutti, A. Carniel, G. Cautero, A. Fabris, A. Gambitta, D. Giuressi, G. Loda, M. Lonza, F. Mazzolini, G. Mian, N. Pangos, R. Sergo, V. Smaluk, R. Tommasini, L. Tosi, L. Zambon, M. Dehler, and R. Ursic, in Proceedings of the DIPAC2001, Grenoble (ESRF, Grenoble, 2001), http:// www.esrf.fr/conferences/DIPAC/ DIPAC2001Proceedings.html

[6] B. W. Zotter and S. A. Kheifets, Impedances and Wakes in High-Energy Particle Accelerators (World Scientific, Singapore, 1998).

[7] E. Keil and W. Schnell, CERN Report No. ISR-TH-RF 69/48, 1969.

[8] A. Chao, Physics of Collective Beam Instabilities in High Energy Accelerators (John Wiley and Sons, Inc., New York, 1993).

[9] V. Kiselev and V. Smaluk, in Proceedings of the DIPAC99, Chester (CLRC Daresbury Laboratory, 1999), http:// srs.dl.ac.uk/dipac/proceedings.pdf

[10] V. Kiselev, V. Smaluk, and V. Zorin, in Proceedings of the PAC-2001, Chicago (IEEE, Piscataway, NJ, 2001).

[11] G.V. Stupakov, in Proceedings of the PAC-2001, Chicago (Ref. [10]).

[12] L. Tosi, V. Smaluk, E. Karantzoulis, in Proceedings of the EPAC-2002, Paris (EPS-IGA/CERN, Geneva, 2002).

[13] L. Tosi, V. Smaluk, D. Bulfone, E. Karantzoulis, and M. Lonza, in Proceedings of the PAC-2001, Chicago (Ref. [10]).

[14] J. L. Revol, R. Nagaoka, P. Kernel, E. Karantzoulis, and L. Tosi, in Proceedings of the EPAC-2000, Vienna (CERN, Geneva, 2000). 